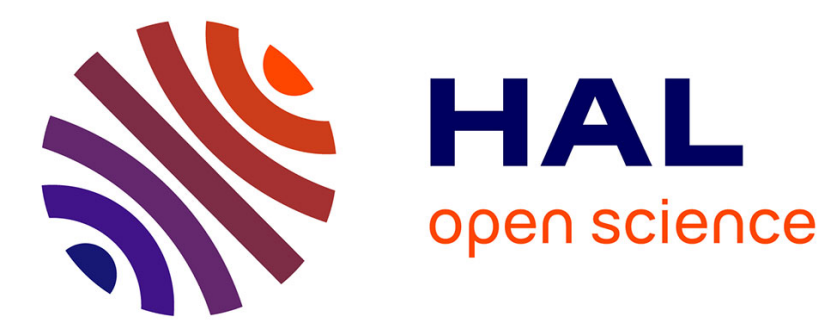

\title{
Laser micro- and nanostructuring using femtosecond Bessel beams
}

\author{
M.K. Bhuyan, F. Courvoisier, H.S. Phing, O. Jedrkiewicz, S. Recchia, P. Di
} Trapani, J.M. Dudley

\section{- To cite this version:}

M.K. Bhuyan, F. Courvoisier, H.S. Phing, O. Jedrkiewicz, S. Recchia, et al.. Laser micro- and nanostructuring using femtosecond Bessel beams. The European Physical Journal. Special Topics, 2011, 199 (1), pp.101-110. 10.1140/epjst/e2011-01506-0 . hal-00661745

\section{HAL Id: hal-00661745 \\ https://hal.science/hal-00661745}

Submitted on 22 Apr 2021

HAL is a multi-disciplinary open access archive for the deposit and dissemination of scientific research documents, whether they are published or not. The documents may come from teaching and research institutions in France or abroad, or from public or private research centers.
L'archive ouverte pluridisciplinaire HAL, est destinée au dépôt et à la diffusion de documents scientifiques de niveau recherche, publiés ou non, émanant des établissements d'enseignement et de recherche français ou étrangers, des laboratoires publics ou privés. 


\section{Laser micro- and nanostructuring using femtosecond Bessel beams}

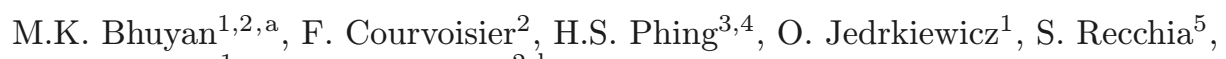
P. Di Trapani ${ }^{1}$, and J.M. Dudley ${ }^{2, b}$

1 CNISM and Dipartimento di Fisica e Matematica, Università dell'Insubria, via Valleggio 11, 22100 Como, Italy

${ }^{2}$ Département d'Optique P. M. Duffieux, Institut FEMTO-ST, UMR 6174, CNRS - Université de Franche-Comté, 16 route de Gray, 25030 Besançon Cedex, France

${ }^{3}$ INRS-EMT, University of Quebec, 1650, Boul. Lionel-Boulet, Varennes, Quebec, Canada J3X1S2

${ }^{4}$ Nanophotonics Research Alliance, Universiti Teknologi Malaysia, 81310 Skudai, Johor, Malaysia

5 Dipartimento di Scienze Chimiche e Ambientali, Università dell'Insubria, via Valleggio 11, 22100 Como, Italy

Abstract. We report the generation of femtosecond Bessel beams of conical half-angle 26 degrees using an axicon lens and a beam reduc-tion imaging setup. The generated Bessel beams were applied to the micromachining of nanostructures in glass of length up to $100 \mu \mathrm{m}$. The effect of the incident pulse energy on the characteristics of the nano-structures was studied using optical microscopy.

\section{Introduction}

The machining of highly reproducible micro- and nanostructures within transparent dielectric materials is a long-standing challenge for potential applications in many important areas such as photonics, microfluidics, and photonic crystal fabrication $[1,2]$. The use of femtosecond laser processing technology has recently been identified as a convenient and robust response to this challenge, and has been successfully applied to produce a wide range of structures such as waveguides, voids and channels. However, this technology still faces the issue of producing high aspect ratio structures because machining using focussed Gaussian beams suffers from the inevitable diffraction-induced tradeoff between beam waist and longitudinal interaction length. This imposes severe limits on the usefulness of this approach. In this paper, we describe how spatial beam shaping using an axicon lens to generate "diffraction-free" femtosecond Bessel beams overcomes this problem, and allows the convenient fabrication of extended channels.

\footnotetext{
${ }^{a}$ e-mail: manoj.femto@gmail.com

b e-mail: john.dudley@univ-fcomte.fr
} 
It is first instructive to review the basic mechanisms of femtosecond pulse-material interactions. When a femtosecond laser pulse is focused inside a transparent material, the pulse generates a free electron plasma through several different ionization mechanisms. When the density of the free electrons in the plasma exceeds a certain threshold, (typically $10^{19}$ per cubic centimeter [3]), the plasma becomes absorbing and a large amount of laser energy is deposited within the excited area of the material, resulting in optical breakdown. As a result, laser induced structures of micron and sub-micron size can be achieved, with the formation of such structures often attributed to a phenomenon known as a microexplosion $[4,5]$.

The dimensions of such laser-induced structures depend on several parameters such as the focusing geometry, laser energy, laser pulse duration, bandgap of the material, and a wide variety of different structural modifications have been reported. For example, by choosing a suitable focusing geometry, periodic sub-micron voids in glass have been observed [6]. The filamentation of the beam resulting in an increase in the longitudinal extent followed by microexplosions are attributed to the formation of such long periodic sub-micron voids.

Much research has also focussed on the production of long, continuous nanostructures (often termed channels) in glass and sapphire. For example, Kudryashov et al. [7] successfully produced nanochannels up to $1 \mu \mathrm{m}$ long using front-surface machining. Herbstman et al. [8] managed to achieve nanochannels up to $10 \mu \mathrm{m}$ long using both front- and reverse-surface machining techniques. Note here that the above studies were performed using an initial Gaussian beam profile, and filamentation occurring within the material plays a key role in attaining such long channels. However, this filamentation based machining technique is not always convenient or reproducible because the control of the highly nonlinear filamentation process when using an incident Gaussian beam is not straightforward.

It is here where the recent work using Bessel beams has suggested a versatile and powerful solution to this problem to provide a controllable approach to nanochannel formation [9]. Using this technique, and taking advantage of the high degree of stability of Bessel filaments compared to Gaussian filaments [10], long nanochannels up to $43 \mu \mathrm{m}$ have been machined in glass. The current study aims to investigate the processing of nanochannels using a more direct setup compared to that in Ref. [9] using direct axicon-generation of the Bessel beam, and also using shorter pulse durations (38 fs).

\section{Theoretical background}

\subsection{Bessel beam - fundamentals}

Bessel beams, first introduced by Durnin et al. [11], are a special class of beam that possess a narrow bright central region surrounded by a series of concentric rings (see Fig. 1(a)) that (ideally) extend over an infinitely long distance and are thus described as "diffraction-free". The field distribution of such beams can be expressed as follows:

$$
E(r, \phi, z, t)=a_{0} J_{0}\left(k_{r} \cdot r\right) \cdot \exp \left[i\left(k_{z} z-\omega t\right)\right]
$$

where $r, \phi, z$ and $t$ are transverse, azimuthal, longitudinal and time variables respectively. $a_{0}$ is the amplitude of the electric field on the optical axis of Bessel beam. $J_{0}$ is the zeroth order Bessel function of first kind. $\omega$ is the angular frequency of the field. $k_{r}$ and $k_{z}$ (sometimes referred as $k_{\perp}$ and $k_{\|}$) are transverse and longitudinal wave vectors related to $k$ through $k=\sqrt{k_{r}^{2}+k_{z}^{2}}$. The intensity associated with the Bessel beam is proportional to $J_{0}^{2}\left(k_{r} \cdot r\right)$ i.e. independent of the longitudinal component. 


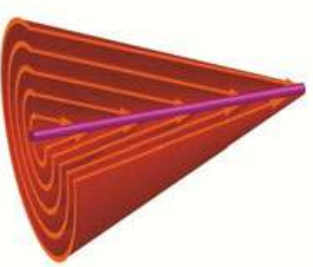

(a)

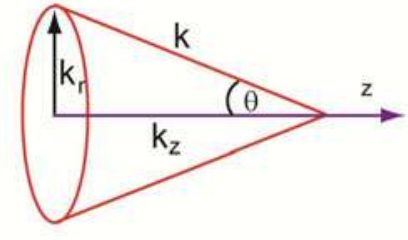

(b)

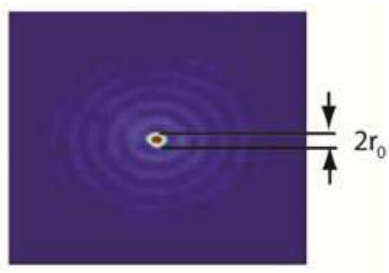

(c)

Fig. 1. Illustrative figures showing Bessel beam characteristics. (a) The conical-flow of energy inwards towards the central lobe of the beam. (b) The relationship between the wave vectors that constitute a non-diffracting Bessel beam. (c) The transverse intensity profile of the Bessel beam. The central core size of the Bessel beam is denoted as $2 r_{0}$.

Taking the Fourier transform of Eq. (1), Bessel beam propagation along the zdirection can be considered as the superposition of an infinite number of plane waves whose wave vectors are distributed on a cone centered on the z-axis. The opening angle (sometimes referred as conical half-angle) $\theta$ of the cone can be expressed as follows:

$$
\theta=\tan ^{-1}\left(\frac{k_{r}}{k_{z}}\right)
$$

Here, $k_{r}$ and $k_{z}$ are expressed as follows: $k_{r}=k \sin \theta$ and $k_{z}=k \cos \theta$ (see Fig. 1(b)).

\subsection{Bessel beam generation}

An ideal Bessel beam as described above contains infinite energy, and hence is not possible to generate experimentally. However, under a finite energy approximation, the experimental generation of Bessel beams over finite distances have been demonstrated. Durnin et al. first reported Bessel beam generation through experiments using an annular slit placed at the back focal plane of a converging lens illuminated by a plane wave. Although this technique is very simple, it suffers from the following drawbacks: (a) Oscillation in the on-axis intensity of the Bessel beam and (b) high throughput loss i.e. most of the incident laser beam is blocked by the slit, allowing light only through the annular section. Several other methods of generation have also been demonstrated. For example, Bessel beams were generated using axicon lenses [12,13], holograms [14] and spatial light modulators $[15,16]$. Here, we discuss the method of generation of Bessel beam using axicon lens in detail.

An illustrative figure of the axicon-based Bessel beam generation technique is shown in Fig. 2. When a collimated monochromatic Gaussian laser beam with a flat phase front is normally-incident on an axicon lens of refractive index $n_{a x}$ and base angle $\alpha$, two portions of the beam deviate towards the main axis i.e. along the propagation direction with an angle $\theta$ and overlap resulting in an interference effect. It is this interference that yields the Bessel beam characteristics. With only geometrical optics considerations, Snell's law at the inclined face of an axicon lens gives rise to the following expressions:

$$
\theta=\arcsin \left(n_{a x} \sin \alpha\right)-\alpha .
$$

For a Gaussian beam of half-width $w_{0}$, the axicon lens produces a Bessel beam with depth of focus $z_{\max }$, defined as follows:

$$
z_{\max } \approx w_{0} \frac{k}{k_{r}} \approx \frac{w_{0}}{\theta} .
$$




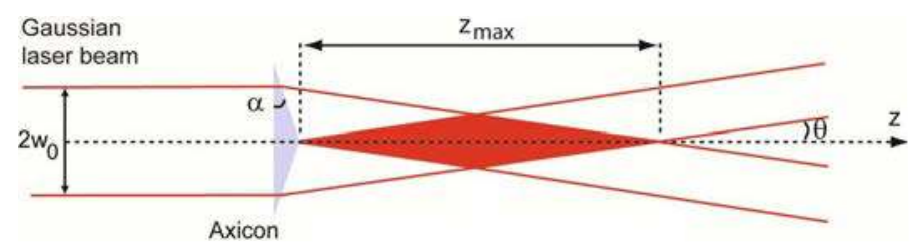

Fig. 2. An illustrative figure showing the generation of Bessel beam from a Gaussian beam of waist $w_{0}$ using an axicon lens of base angle $\alpha$ is shown.

The size of the central core (i.e. the radial distance between the first intensity minima around the core of the Bessel beam) can be expressed as $2 r_{0}=\frac{4.81}{k_{r}}$ (see Fig. 1(c)). We note that the depth of focus of a Bessel beam is several orders of magnitude larger than the Rayleigh length associated with Gaussian beams of the same spot size.

The mathematical description of a Bessel beam generated by an axicon is usually based on the Fresnel diffraction integral evaluated under stationary phase method [17]. The analytical expression derived in this way is a good approximation in the area close to the beam axis. Jarutis et al. [18] derived an analytical expression that fits well the Fresnel diffraction integral almost over the whole pattern of Bessel beam produced by an axicon illuminated by appropriate Laguerre-Gaussian (LG) beam. The onaxis intensity $I(z)$ produced by an axicon lens illuminated by a Gaussian beam at a distance $\mathrm{z}$ from the beam onset i.e. axicon tip can be expressed as follows:

$$
I(z)=\frac{8 \pi P_{0} z \sin ^{2} \theta}{\lambda w_{0}^{2}} \exp \left[-2\left(\frac{z \sin \theta}{w_{0}}\right)^{2}\right]
$$

where $P_{0}$ is the peak power of the laser beam incident on the axicon lens.

\section{Experimental setup}

The experimental setup used for the nanostructuring of transparent materials using Bessel beam is shown in Fig. 3. The technical details of all the optical elements and systems that are used for our experiments are presented in Table 1. The setup is based on a $38 \mathrm{fs}$ Ti:Sapphire laser system. The energy of the laser pulse is controlled using an attenuator consisting of a combination of half-wave plate and a Glan-laser type polarizer. The polarization of the beam is converted into circular polarization using a quarter-wave plate. The Gaussian beam is then transformed into a Bessel beam using a 1 degree axicon lens, as explained in Section 2.2. The generated Bessel beam is then demagnified using a telescopic arrangement consisting of a plano-convex lens of focal length $200 \mathrm{~mm}$ and a microscope objective lens (x50 numerical aperture 0.8) with an effective focal length of $3.6 \mathrm{~mm}$. The resultant Bessel beam (referred hereafter as micro-Bessel beam) that is generated at the focal plane of the microscope objective lens is used for nanostructuring of glass samples. Since the micro-Bessel beam starts from the focal plane of the microscope objective lens, this focal plane is treated as a virtual axicon lens.

In order to characterize the micro-Bessel beam, we used an imaging setup consisting of a microscope objective lens (identical to the one used for the generation of the Bessel beam) mounted on a translation stage. The focal plane of this microscope objective lens is conjugated to a camera using a plano-convex lens of focal length $150 \mathrm{~mm}$. The magnification of the imaging setup was measured to be 42.6 . The transverse sections of the micro-Bessel beam along the propagation direction are recorded 
Table 1. Technical details of the optical components or systems used in the machining setup. Notations: $\lambda$ : wavelength, $\tau$ : pulse duration, LRR: laser repetition rate, $w_{0}$ : Beam waist of laser beam, f: focal length, D: diameter of the optical component, $\alpha$ : base angle of the axicon lens, $n_{a x}$ : refractive index of axicon lens, UVFS: UV fused silica.

\begin{tabular}{|c|c|c|}
\hline No. & Components & Technical details \\
\hline 1 & $\begin{array}{l}\text { Ti:Sapphire laser system } \\
\text { (Amplitude, Inc.) }\end{array}$ & $\lambda=800 \mathrm{~nm}, \tau=38 \mathrm{fs}, \mathrm{LRR}=20 \mathrm{~Hz}, w_{0}=7 \mathrm{~mm}$ \\
\hline 2 & Axicon (Altechna Inc.) & $\mathrm{D}=2$ inch, $\alpha=1 \mathrm{deg}, n_{a x}=1.45, \mathrm{UVFS}$ \\
\hline 3 & $\begin{array}{l}\text { Translation stages } \\
\text { (Aerotech, Inc.) }\end{array}$ & 3-axis, Resolution: $10 \mathrm{~nm}$ \\
\hline 4 & Sample (Corning inc.) & $150 \mu \mathrm{m}$ thick Glass slides \\
\hline 5 & Camera (uEye Inc.) & Resolution $=1.3$ Megapixel, Pixel pitch $=5.3 \mu \mathrm{m}$ \\
\hline 6 & Microscope objective lens & $\times 50$, NA $0.8, \mathrm{f}=3.6 \mathrm{~mm}$ \\
\hline
\end{tabular}

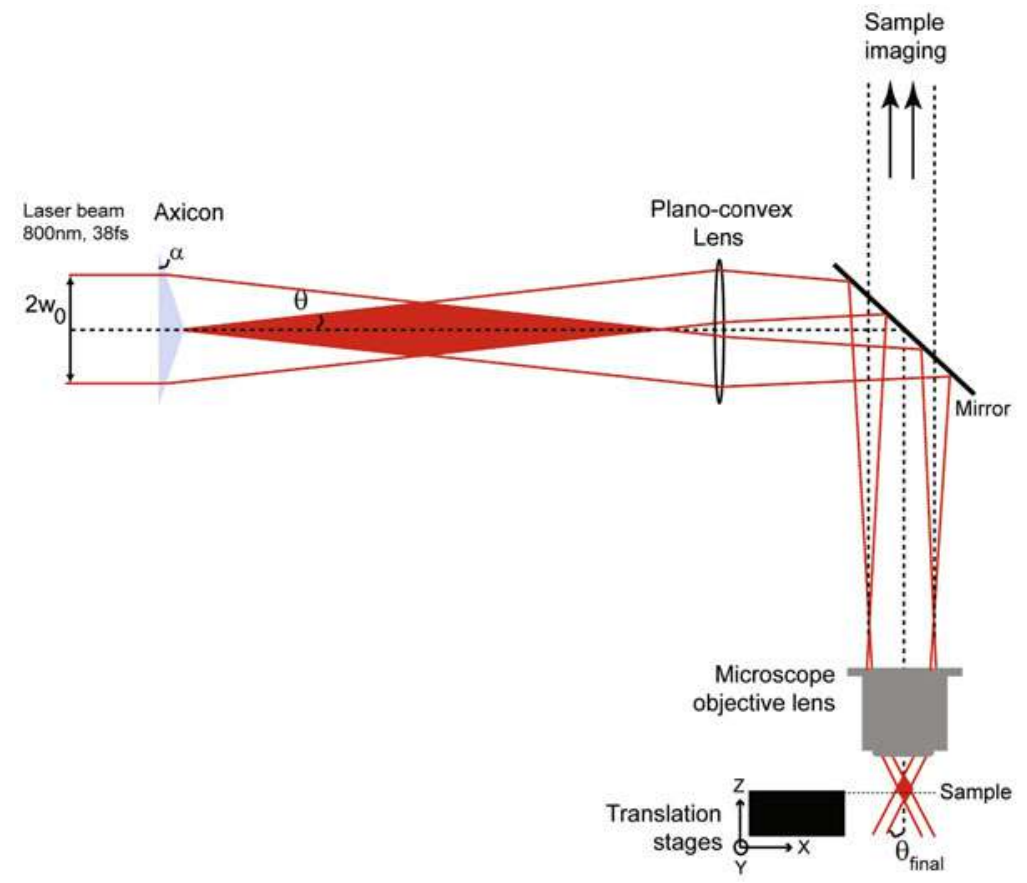

Fig. 3. Schematic diagram of the laser machining setup. The Gaussian laser pulses coming from a Ti:Sapphire laser system are first converted into Bessel beams and further demagnified using a Plano-convex lens and a microscope objective lens. The demagnified Bessel beams are used for the nanostructuring of glass samples mounted on a 3-axis translation stages. Samples are observed in-situ from top using an imaging setup (not shown in this figure).

by translating the microscope objective lens by $1 \mu \mathrm{m}$ steps. These images are numerically processed to measure the beam central core size and longitudinal extent.

The micro-Bessel beam was then used for the machining of nanostructures in Corning 0211 glass samples with thickness $150 \mu \mathrm{m}$ placed on a sample holder mounted on 3-axis motorized translation stages. An electronic shutter was utilized to control the number of pulses used for machining. Note here that, for the present experiments, we use only one laser pulse to create a single nanostructure in glass. The sample surface was monitored using an imaging setup, which allowed the control and correction of the sample tilt to within $1 \mathrm{mrad}$. 


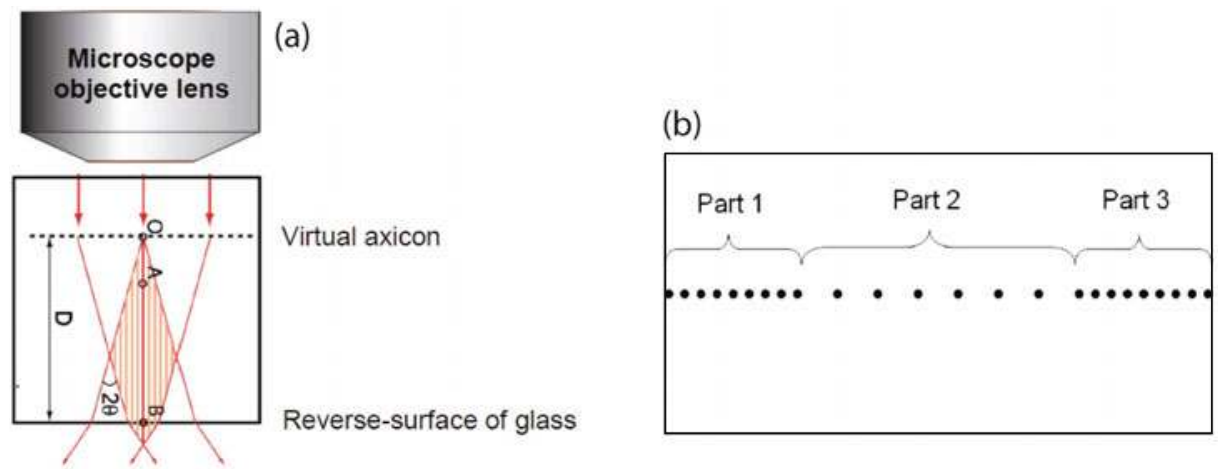

Fig. 4. Machining geometry. (a) A simplified diagram of the Bessel beam structuring setup is shown where the sample is placed in the Bessel beam for nanostructuring. The focal plane of the microscope objective lens is treated as a virtual axicon lens; the distance between the reverse-surface of sample and the virtual axicon is denoted as $\mathrm{D}$, an important experimental parameter for nanostructuring technique. (b) Typical machining pattern: Part 1 and Part 3 represent an array of closely packed nanostructures machined to support the cleaving of the sample. Part 2 i.e. the region of interest represents an array of nanostructures with large separation, typically 15 to $25 \mu \mathrm{m}$, which we would like to characterize after cleaving along their length.

Figure 4(a) is an illustrative figure showing the machining geometry, correlating the beam position with respect to the reverse-surface of the sample. Note here that we define an experimental parameter $\mathrm{D}$, the distance between the virtual axicon and the reverse-surface of the sample. In our experiments, the structures are machined on the reverse-surface of the sample and we vary the parameter D by vertically translating the sample.

In our experiments we used only single laser shots for machining, translating the sample in the y-direction after every shot. The nanostructures under investigation were produced at the center of the sample (Part 2 as illustrated in Fig. 4(b)). In this part, the separation of the structures is in the range $15-25 \mu \mathrm{m}$ to avoid distortions by adjacent - previously produced - nanostructures. In order to cleave the sample through the structures, we produce a planar stress along the whole sample by machining a line of identical nanostructures separated by $1 \mu \mathrm{m}$ on both sides of the central region (part 1 and part 3 ).

Once the machining process is finished, the sample is removed from the sample holder and mechanical stress is applied on either side of the nanostructure. This results into 2 replicas of the sample containing the nanostructures. The surface of the sample containing the nanostructures are then observed under an optical microscope for preliminary investigations. In this work, measurements of the diameters of the nanostructures are limited by the resolution of the optical microscope, but scanning electron microscope images can be readily performed if needed [9]. But we note that microscopic inspection as performed here can be sufficient to determine and optimise many of the experimental parameters and to perform studies of channel length dependence on energy.

\section{Results and discussion}

Here, we present the experimental generation of micro-Bessel beams of very high conical half-angle and their subsequent applications to nanostructuring of $150 \mu \mathrm{m}$ thick borosilicate glass sample (Corning 0211). 


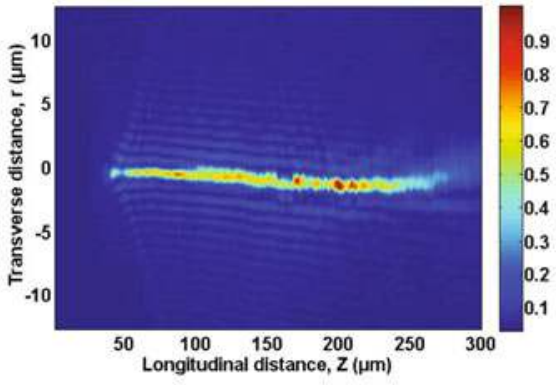

(a)

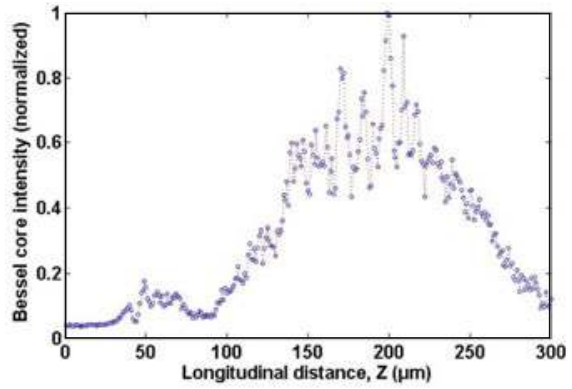

(b)

Fig. 5. (a) The longitudinal profile of the Bessel beam reconstructed by a series of transverse cross section images and (b) the normalized intensity distribution of the central core of the Bessel beam along the propagation axis (z).

\subsection{Generation of Bessel Beam}

As discussed in Section 2.2, the transverse beam profiles of the Bessel beam along the propagation direction are recorded using a camera with an incremental step of $1 \mu \mathrm{m}$. From this series of transverse beam profiles, we reconstructed the whole beam. Fig. 5(a) shows the longitudinal cross section of the micro-Bessel beam along the propagation axis $(\mathrm{z})$. As we see from these figures, a Bessel beam of long and narrow central core is produced by the constructive interference of the Gaussian beam with the help of an axicon lens and fringes (rings) with lower intensities surrounding the beam core are observed.

The central core size $\left(2 \mathrm{r}_{0}\right)$ is measured to be $880 \mathrm{~nm}$ and the conical half angle $\theta$ is 26 degrees. For useful comparison with previous studies, microchannels [19] and nanochannels [9] have been machined using Bessel beam of similar core sizes.

Figure 5(b) shows the axial intensity distribution of the micro-Bessel beam extracted from the longitudinal beam profile, as shown in Fig. 5(a). The longitudinal extent at full width at half maximum position is measured to be $145 \mu \mathrm{m}$, that is almost one order of magnitude larger than the micro-Bessel beam used in Ref. [9]. Note here that the longitudinal extent of the Bessel beam can be varied by controlling the input beam (Gaussian) size through an adjustable aperture; it was also verified through experiments.

\subsection{Bessel beam nanostructuring in glass}

In this section, we present results studying the dependence of the characteristics of nanostructures in glass on energy per pulse. We machined nanostructures in the reverse-surface of the glass sample in the energy range $4-18 \mu \mathrm{J}$ for a fixed parameter $\mathrm{D}=105 \mu \mathrm{m}$. Note here that the criterion of choosing the value of $D$ is that the intensity of the central core of the Bessel beam at the reverse-surface of the sample (point $\mathrm{B}$ of Fig. 4(a)) must exceed a threshold value, typically $10^{13}-10^{14} \mathrm{~W} / \mathrm{cm}^{2}$ [9]. The length of the nanostructure machined with this configuration would be the distance between point A and B of Fig. 4(a), where the Bessel core intensity exceeds the threshold value. Note here that the threshold value for channel and index modification are essentially different. As discussed in Section 3, the results are analyzed on the basis of post-processing characterization of cleaved nanostructures using optical microscopy. Here, our comments are restricted only to the length of the nanostructures. 


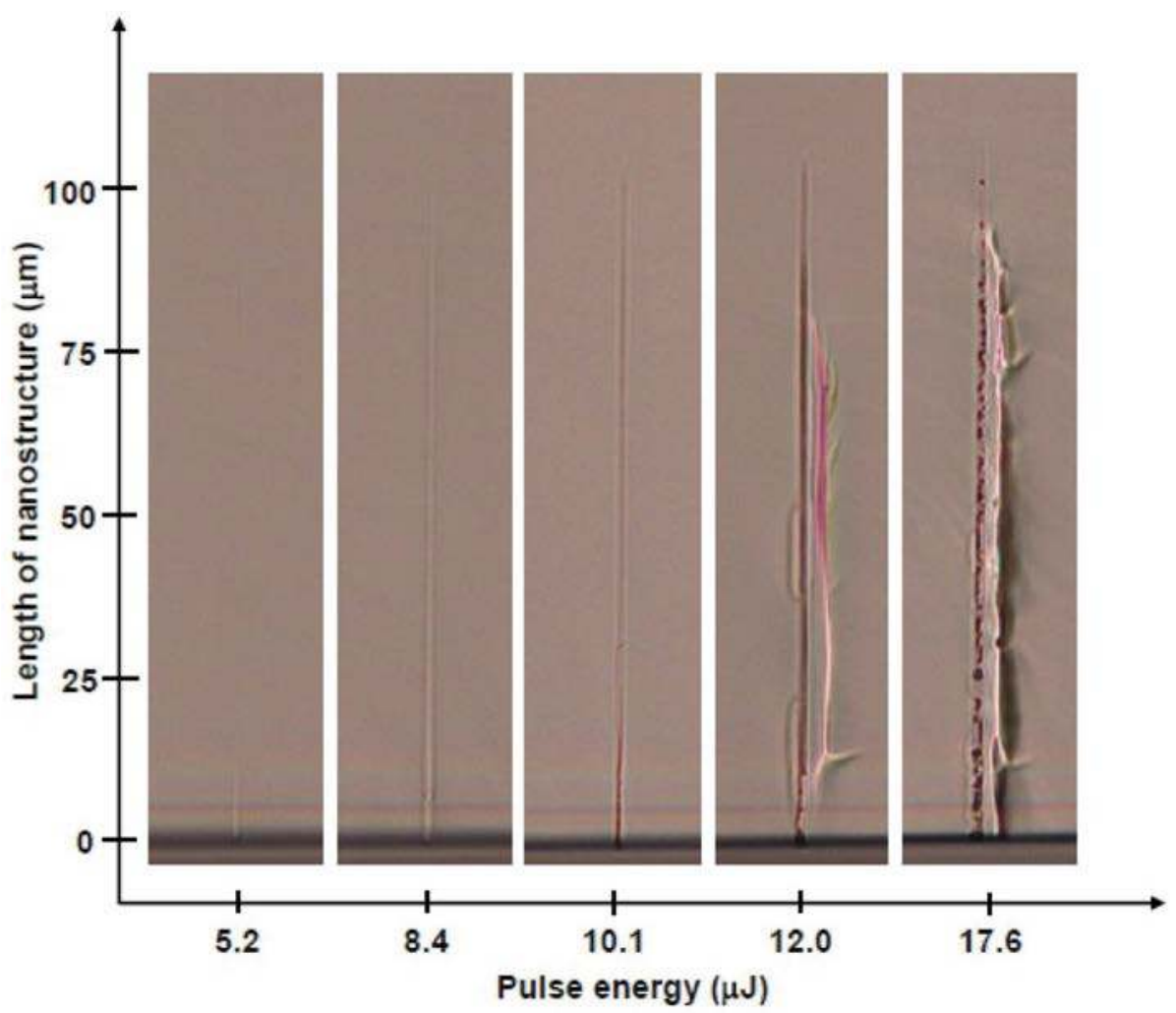

Fig. 6. Optical microscopy images of the laser induced nanostructures in the reverse-surface of glass machined with Bessel beam. The beam direction is from top to bottom surface of the sample.

Figure 6 illustrates the channel characteristics as a function of the energy of the Bessel beam. It is observed that the threshold energy of $5.2 \mu \mathrm{J}$ is required to induce damage on the reverse-surface of the glass. Further, we observed long nanostructures when machined with $10 \mu \mathrm{J}$ pulses. The contrast of the microscopic image indicates that there are two different types of features embedded in a single nanostructure. We attribute the feature attached to the exit surface of the sample as a channel and the remaining portion of the nanostructure as an index modified region. However, for a laser energy of $12 \mu \mathrm{J}$, machined nanostructures are uniform. We attribute this feature as a channel, which is consistent with the results shown in Ref. [9]. Figure 7 shows a scanning electron microscopy image of a typical structure produced in this energy regime, confirming the existence of channels. We attribute the mechanism of the formation of such a long, uniform nanostructure to the stationarity of Bessel beams [10] at ablation-level intensity and to the hydrodynamic material removal process [20]. Experimentally, we also observed that the structures are very consistent from laser shot-to-shot signifying the stationarity of the Bessel beams. In addition, we note that irregular shapes are visible aside the channels. These shapes vary from one sample to another and are due to sample cleaving.

For higher laser energy i.e. for $17.6 \mu \mathrm{J}$ pulses, the damage is more pertinent and the nanostructures are distorted. These structures can be usefully compared to the results obtained by Kanehira et al. [6], where they observed periodic nanovoids in glass. They attributed the mechanism responsible for the creation of such features to 


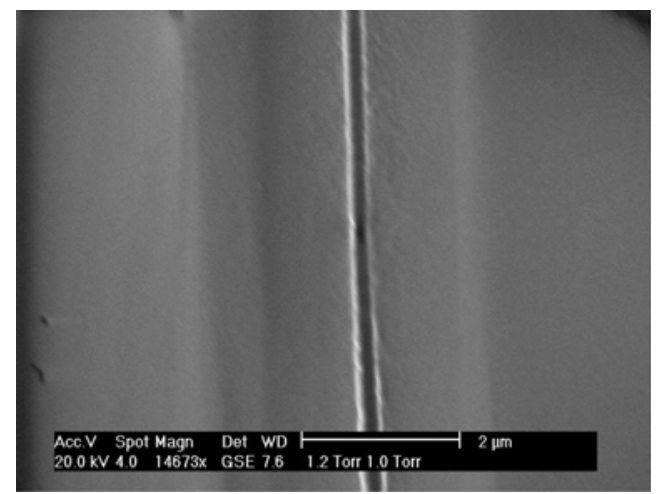

Fig. 7. Scanning electron microscopy image of the central part of a laser induced nanostructure in the reverse-surface of glass machined with Bessel beam.

a combinatorial process i.e. filamentation followed by micro-explosion in the bulk of the sample. We note that this regime was not observed in previous studies with pulse duration of $230 \mathrm{fs}$ [9] and that shorter pulse duration enhance nonlinear dynamical effects.

\section{Conclusions}

It is demonstrated that Bessel beams generated by femtosecond laser using an axicon lens enable us to machine long and narrow nanostructures in glass effectively, opening an alternative path for high aspect ratio (length/diameter) microfluidic and photonic device fabrication. We have successfully generated Bessel beam extending over $145 \mu \mathrm{m}$ in the longitudinal direction and central core size of $880 \mathrm{~nm}$ using 1 degree axicon lens. By using this Bessel beam, we have machined $100 \mu \mathrm{m}$ long nanostructures in glass using laser energy of $12 \mu \mathrm{J}$. Our results also identified the threshold laser energy required for the fabrication of either channel or index modification in glass with possible application in the area of photonics and nanofluidics.

The experiment has been performed in the context of STELLA, the School for Training in Experiments with Lasers and Laser Applications, held at the Insubria University in Como from June 20 to July 82011 (see www.stella-school.eu). The Authors wish to acknowledge CARIPLO, UNIVERCOMO and Banca del Monte di Lombardia Foundations for having financed the project. The Authors would also like to acknowledge the STELLA-school students X. Zambrana-Puyalto and O. de J. Ramírez-Góngora for their support in the experiment.

\section{References}

1. Gattass, et al., Nat. Phot. 2, 219 (2008)

2. Valle, et al., J. Opt. A: Pure Appl. Opt. 11, 013001 (2009)

3. Sun, et al., Appl. Phys. A 88, 285 (2007)

4. Glezer, et al., Appl. Phys. Lett. 71, 882 (1997)

5. Gamaly, et al., Phys. Rev. B 73, 214101 (2006)

6. Kanehira, et al., Nano Lett. 5, 1591 (2005)

7. Kudryashov, et al., Appl. Phys. Lett. 91, 141111 (2007)

8. Herbstman, et al., Opt. Exp. 18, 16840 (2010)

9. Bhuyan, et al., Appl. Phys. Lett. 97, 081102 (2010) 
10. Polesana, et al., Phys. Rev. A 77, 043814 (2008)

11. Durnin, et al., Phys. Rev. Lett. 58, 1499 (1987)

12. J.H. McLeod, J. Opt. Soc. America 50, 166 (1960)

13. Herman, et al., J. Opt. Soc. America A 8, 932 (1991)

14. Turunen, et al., Appl. Opt. 27, 3959 (1988)

15. Vasara, et al., J. Opt. Soc. America A 6, 1748 (1989)

16. Davis, et al., Appl. Opt. 35, 599 (1996)

17. Arlt, et al., Phys. Rev. A 63, 063602 (2001)

18. Jarutis, et al., Opt. Commun. 184, 105 (2000)

19. Bhuyan, et al., Opt. Exp. 18, 566 (2010)

20. Juodkazis, et al., Phys. Rev. Lett. 96, 166101 (2006) 